\title{
Reductions of Erythrocyte Membrane Viscoelastic Coefficients Reflect Spectrin Deficiencies in Hereditary Spherocytosis
}

\author{
Richard E. Waugh* and Peter Agre ${ }^{*}$ \\ ${ }^{*}$ Department of Biophysics, University of Rochester School of Medicine and Dentistry, Rochester, New York $14642 ;{ }^{\ddagger}$ Department of \\ Medicine, The Johns Hopkins University School of Medicine, Baltimore, Maryland 21205
}

\begin{abstract}
Hereditary spherocytosis is a common hemolytic anemia associated with deficiencies in spectrin, the principal structural protein of the erythrocyte membrane-skeleton. We have examined 20 different individuals from 10 spherocytosis kindreds and 2 elliptocytosis kindreds to determine the effects of different levels of spectrin deficiency on the viscoelastic properties of the erythrocyte membrane. Micropipettes were used to perform single-cell micromechanical measurements of $\sim 1,000$ individual cells to determine the membrane elastic shear modulus, the apparent membrane bending stiffness, and whole cell recovery time constant for the different cell populations. The membrane viscosity was calculated by the product of the shear modulus and the recovery time constant. Results show correlation between the fractional reduction in shear modulus and the fractional reduction in spectrin content (determined by spectrin radioimmunoassay) and spectrin density (determined by the ratios of spectrin to band 3 on electrophoresis gels) suggesting that membrane shear elasticity is directly proportional to the surface density of spectrin on the membrane $(P<0.001)$. The apparent membrane bending stiffness is also reduced in proportion to the density of spectrin $(P<0.001)$. The membrane viscosity is reduced relative to control $(P<0.001)$, but the nature of the relationship between spectrin density and membrane viscosity is less clearly defined. These studies document striking relationships between partial deficiencies of erythrocyte spectrin and specific viscoelastic properties of the mutant membranes.
\end{abstract}

\section{Introduction}

The pathophysiology of hereditary spherocytosis (HS) ${ }^{1}$ has been the subject of clinical and biophysical investigation for decades (see Weed [1] and Becker and Lux [2] for reviews). Early investigators recognized that the basis of the disorder resided in the red cell itself, and that the cells had decreased surface-to-volume ratio (S/V), probably due to loss of surface

Address reprint requests to Dr. Waugh, Box BPHYS, University of Rochester School of Medicine and Dentistry, 601 Elmwood Avenue, Rochester, NY 14642.

Received for publication 21 January 1987 and in revised form 27 July 1987.

1. Abbreviations used in this paper: HS, hereditary spherocytosis; $\mathrm{MCHC}$, mean cellular hemoglobin concentration; $\mathrm{S} / \mathrm{V}$, surface to volume ratio.

J. Clin. Invest.

(C) The American Society for Clinical Investigation, Inc.

0021-9738/88/01/0133/09 \$2.00

Volume 81, January 1988, 133-141 area. Further delineation of the underlying mechanism in the disorder has been possible because of advances occurring over the past decade both in our knowledge of the molecular composition and organization of the erythrocyte membrane and in our understanding and characterization of the mechanical behavior of the membrane.

The membrane skeleton is a protein "scaffolding" at the cytoplasmic surface of the membrane bilayer that gives the erythrocyte its elastic character and contributes to its stability (for review see Bennett [3]). The membrane-skeleton consists primarily of spectrin, a heterodimer of alpha and beta subunits that associate head-to-head with other spectrin heterodimers to form tetramers and higher order oligomers. These spectrin oligomers are in turn cross-linked by tail end associations with short actin protofilaments and protein 4.1 forming a network. This network is held to the bilayer by associations between spectrin, ankyrin and the integral protein, band 3 , and by associations between band 4.1 and a subset of the integral proteins, the glycophorins. Recently, it has been demonstrated that partial deficiency of spectrin is a general feature of many different forms of HS (4) and that the magnitude of the deficiency correlates with the clinical severity of the anemia (5).

Concurrent with the growth in understanding of the biochemical aspects of membrane-skeletal structure, the understanding and ability to evaluate the mechanical properties of the erythrocyte membrane have grown in sophistication. (See Evans and Skalak [6] and Hochmuth and Waugh [7] for reviews). The membrane has a large resistance to changes in surface area so that under physiologic conditions the membrane area can be considered constant. (This does not exclude the possibility that cellular area can be reduced by loss of membrane fragments, microvesiculation or chemical degradation.) The membrane also exhibits shear rigidity, an elastic resistance to extensional deformations of the surface plane at constant area, that is characterized by the membrane shear modulus $(\mu)$. The elastic resistance of the membrane to changes in curvature is characterized by the bending stiffness (B). These elastic coefficients can be used to predict membrane behavior under static (stationary) loads. To predict membrane behavior in dynamic situations an additional coefficient, the membrane viscosity $(\eta)$, is needed. While the actual dynamic behavior of the membrane is complex $(8,9)$, a single constant coefficient relating membrane force resultants to the rate of surface deformation is adequate for characterizing the membrane behavior under physiologic conditions.

The identification and characterization of molecular abnormalities in hemolytic anemias coupled with the ability to accurately measure membrane mechanical properties has provided important opportunities to learn more about the structure-function relationship of the red cell membrane skeleton. In a previous report (10), the force required to form membrane strands (tethers) from red cells was measured for a number of 
patients with different molecular abnormalities. Although the tethering force was consistently lower for the abnormal membranes compared to control, the extent of the mechanical dysfunction did not correlate well with the biochemical characterization of the membrane defect, suggesting that the biochemical characterizations may have been incomplete, and that other yet unidentified molecular lesions were responsible for the mechanical abnormality. Indeed, subsequent biochemical analyses identified spectrin deficiencies with abnormal spectrin variants in patients with defective spectrin-4.1 linkages (2) and patients with reduced ankyrin binding sites (11). In a preliminary study of eight individuals, six of whom were known to be spectrin deficient (12), a reduction in membrane shear modulus was observed that was similar to the apparent reduction in the surface density of spectrin on the membrane (as indicated by the spectrin/band 3 ratio). In the present work we have taken advantage of the extensive characterization of absolute spectrin content (determined by radioimmunoassay) and spectrin density (spectrin:band 3 ratios on electrophoretic gels) in 12 different kindreds (5) to examine the relationship between spectrin deficiency and the viscoelastic coefficients that are important for characterizing and predicting the cellular behavior under physiologic conditions.

\section{Methods}

Blood samples were obtained by venipuncture, anticoagulated with acid-citrate-dextrose, and shipped on ice via overnight carrier to Dr. Waugh for determination of mechanical coefficients and Dr. Agre for biochemical analyses. All measurements were performed within $48 \mathrm{~h}$ of phlebotomy. Measurements were obtained on blood from $18 \mathrm{HS}$ patients from 10 different kindreds, two heterozygous patients from a family with ovalocytosis and an elongated protein 4.1 variant $(13,14)$ one patient with elliptocytosis and an $\alpha \mathrm{IT} 68$ spectrin variant (11), and one normal individual who had undergone splenectomy after trauma (in addition to matched normal controls for each experiment). The patients are listed in Table I, along with pertinent clinical and genetic information.

\section{Biochemical measurements}

Spectrin density. The normalized spectrin/band 3 ratios were obtained in conjunction with a recent report (5), with the exception of patient 16, whose data appeared in a separate report (2). After separation from other cells, red cells were lysed and washed repeatedly until white membranes were obtained (15). SDS-PAGE was performed according to the methods of Fairbanks et al. (16) using 3.5-17\% nonlinear acrylamide gradient slabs. Membranes were dissolved in SDS (1\%) and $\sim 25 \mu \mathrm{g}$ of protein was applied to the gel and electrophoresed at $250 \mathrm{~V}$ for $3 \mathrm{~h}$. The gels were stained in Coomassie Brilliant Blue R250 overnight, then destained until the background became colorless. The appropriate bands were cut from the gel and eluted overnight in $1.0 \mathrm{ml}$ of $25 \%$ (vol/vol) pyridine in water (17), and absorbance was measured at $605 \mathrm{~nm}$. The ratio of the absorbance of spectrin to the absorbance of band 3 was taken as the spectrin/band 3 ratio. For normal cells the ratio was $\sim 1.0$. Five determinations were made for each patient and control, and the ratio for the patient divided by the ratio for the control gives the "normalized spectrin/band 3 ratio."

Spectrin content. The spectrin radioimmunoassay measured competition between spectrin in detergent solubilized erythrocytes and ${ }^{125}$ I-labeled spectrin for a limiting number of sites on immunoadsorbents as described (4). Human spectrin was purified (15) and ${ }^{125}$ I-labeled to approximately $2 \times 10^{7} \mathrm{cpm} / \mu \mathrm{g}(18)$. The immunoadsorbent was prepared by incubating formalin fixed protein A-bearing Staphylococci with high titer serum from rabbits immunized with human spectrin (19). Patient and control blood specimens were washed three times in PBS, diluted to hematocrits of $10 \%$, and exact cell counts were established by a Coulter counter (Coulter Instruments, Inc., Hialeah, FL). Aliquots were solubilized by incubation in $1 \% \mathrm{SDS}$ at $60^{\circ} \mathrm{C}$ for 10 min and serially diluted into radioimmunoassay buffer $(150 \mathrm{mM}$ $\mathrm{NaCl}, 10 \mathrm{mM} \mathrm{NaPO}$, pH 7.5, $1 \mathrm{mM} \mathrm{NaEDTA}, 1 \mathrm{mM} \mathrm{NaN} \mathrm{m}_{3}, 1 \%$ (vol/vol) Triton X-100, $1 \mathrm{mg} / \mathrm{ml}$ bovine serum albumin, $5 \mu \mathrm{g} / \mathrm{ml}$ pancreatic trypsin inhibitor).

The assay was performed in $12 \times 75$ polystyrene tubes in a $0.2-\mathrm{ml}$ volume of radioimmunoassay buffer containing ${ }^{125} \mathrm{I}$-labeled spectrin $(100,000 \mathrm{cpm})$, dilutions of detergent solubilized erythrocytes (varying from 1:40 to $1: 12,000)$, and immunoadsorbent $(0.5 \%$ vol/vol Staphylococcus suspension with sufficient capacity to precipitate $50 \%$ of the ${ }^{125}$ I-labeled spectrin). After shaking overnight at $4^{\circ} \mathrm{C}$, the immunoprecipitates were washed twice in $2 \mathrm{M}$ urea, $1 \%$ Triton X-100, $0.1 \mathrm{M}$ glycine, and counted in a gamma counter. Spectrin content for each patient was compared to that of the control by linear regression of displacement profiles where immunoprecipitated ${ }^{125} \mathrm{I}$-labeled spectrin was plotted against the number of erythrocytes calculated for each dilution.

Mechanical measurements. Upon receipt, cells were separated from the plasma by centrifugation and resuspended in PBS (125 mM $\mathrm{NaCl}, 25 \mathrm{mM} \mathrm{Na} \mathrm{HPO}_{4}, 6.2 \mathrm{mM} \mathrm{KH}_{2} \mathrm{PO}_{4}$, and $10 \mu \mathrm{M} \mathrm{NaN}$ ) with serum proteins $(3-5 \mathrm{mg} / \mathrm{ml}$ bovine serum albumin or $2.0 \%(\mathrm{vol} / \mathrm{vol})$ autologous plasma). Before use, all solutions were filtered through $0.2-\mu \mathrm{m}$ filters to remove bacteria and debris.

A dilute cell suspension was placed in a small chamber $(1.0 \times 2.0$ $\times 0.1 \mathrm{~cm})$ consisting of a plastic U-shaped spacer between two glass coverslips. The chamber was sealed with vacuum grease, except at the open side of the "U." A micropipette (0.9-1.2 $\mu \mathrm{m}$ i.d.) was introduced through the open side of the " $U$ " and individual cells were aspirated according to the procedures outlined below. After each cell, the field of view was shifted to a new location, to minimize exposure of the cells to the high-intensity microscope illumination. Exposure of individual cells was limited to $<5 \mathrm{~min}$, and the time cells were allowed to remain in the chamber on the microscope stage was $<45 \mathrm{~min}$. The aspiration of individual cells was observed and recorded via a television camera. A data mixer was used to incorporate a digital readout of the aspiration pressure and time into the videorecording. Experiments were performed at room temperature $\left(20-25^{\circ} \mathrm{C}\right)$.

Shear modulus and creasing pressure measurements. This method is essentially the same as previously described $(20,21)$. Cells were aspirated in the dimple region at an initial suction between 2.0 and 3.0 $\mathrm{mm} \mathrm{H}_{2} \mathrm{O}\left(\sim 200-300 \mathrm{dyn} / \mathrm{cm}^{2}\right)$ (Fig. $1 A$ ). Pressure was controlled by changing the level of a water-filled reservoir with a micrometer, and was monitored with a pressure transducer (Validyne Engineering Corp., Northridge, CA: DP103-12). The suction pressure was increased in five to nine increments until creases were observed to form on the cell surface. The length of the aspirated membrane projection was measured as a function of pressure from the videorecordings. The shear modulus $(\mu)$ was calculated by linear regression to the length, pressure data pairs according to the equation proposed by Chien (8):

$$
\frac{\Delta P \cdot R_{\mathrm{p}}}{\mu}=C_{1}\left(L / R_{\mathrm{p}}\right)+C_{2},
$$

where $C_{1}=2.45, C_{2}=-0.603, \Delta P$ is the aspiration pressure, $R_{\mathrm{p}}$ is the pipette radius, and $L$ is the length of the aspirated projection.

The pressure at which creases first formed in the cell surface was also noted (Fig. $1 B$ ). Evans (22) has derived an approximate relationship between the creasing pressure and the membrane bending stiffness. His analysis is based on membrane stability analysis and contains simplifying assumptions about the membrane geometry and the membrane force resultants. For cells that are geometrically similar and aspirated with the same pipette, the creasing pressure should provide an accurate comparison of the bending stiffness of the two membranes. However, the extent to which geometric differences between cell populations may affect Evans' derived relationship is not known with precision, and differences in creasing pressure observed between popula- 

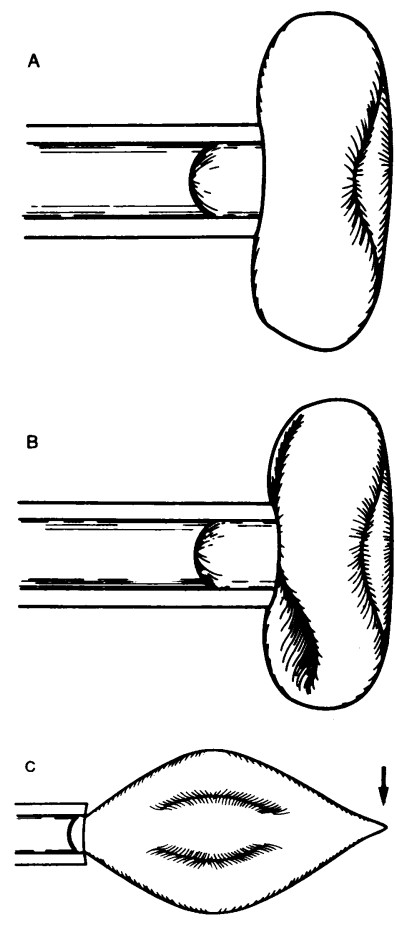
The time constant for the recovery is equal to the ratio of the membrane viscosity to the membrane elastic modulus $\left(\eta=\mu \cdot t_{\mathrm{c}}\right)$.

tions of cells with significantly different geometries should be interpreted cautiously.

Recovery time constant measurements. This procedure has also been described elsewhere $(21,23)$. The bottom coverglass of the cell chamber was pretreated with a dilute, filtered solution of PBS containing autologous plasma $(2.0-6.0 \%, \mathrm{vol} / \mathrm{vol})$ to obtain the desired adhesiveness between the cells and the glass surface. Cells were allowed to settle onto the glass surface and remain undisturbed for 20-30 min during which time they became attached to the glass. A pipette was used to aspirate the cell opposite to the attachment site and stretch the cell into a spindle shape (Fig. $1 C$ ). The cell was released, and the time course of the recovery is recorded. The length-to-width ratio of the cell $(L / W)$ during the recovery is measured as a function of time $(t)$ from the videorecordings. The time constant for the recovery, $t_{\mathrm{c}}$, is obtained by least squares regression to the equation for the recovery of a rectangular membrane strip (23):

$L / W=(L / W)_{\infty} \cdot \frac{X+e^{-t / t_{c}}}{X-e^{-t / t_{c}}}$

where

$X=\frac{(L / W)_{m}+(L / W)_{\infty}}{(L / W)_{m}-(L / W)_{\infty}}$.

The subscript " $\mathrm{m}$ " denotes the maximum length-to-width ratio (at release) and the subscript " $\infty$ " denotes the fully recovered state. The time constant is the ratio of the membrane viscosity $(\eta)$ to the shear modulus $(\mu): t_{\mathrm{c}}=\eta / \mu$. In the regression $(L / W)_{\mathrm{m}}$ is fixed, and $t_{\mathrm{c}}$ and $(L / W)_{\infty}$ are "free" parameters determined by the regression. The membrane viscosity is calculated by taking the product of the shear modulus and the recovery time constant: $\eta=\mu t_{\mathrm{c}}$.

Area and volume measurements. Cells were aspirated into the micropipette at a pressure sufficient to draw the outer portion into a sphere $\left(\sim 6,000 \mathrm{dyn} / \mathrm{cm}^{2}\right)$. The length of the projection, $L$, and the radius of the spherical portion outside the pipette $R_{\mathrm{o}}$ were measured, and the area $A$ and the volume $V$ were calculated according to the following equations:

$$
\begin{aligned}
& A=2 \pi R_{0}\left(R_{0}+\sqrt{R_{0}^{2}-R_{\mathrm{p}}^{2}}\right)+2 \pi R_{\mathrm{p}} L \\
& V=2 / 3 \pi\left[{R_{\mathrm{o}}}^{3}+\left(R_{\mathrm{o}}{ }^{2}+\frac{R_{\mathrm{p}}{ }^{2}}{2}\right) \sqrt{{R_{\mathrm{o}}}^{2}-R_{\mathrm{p}}{ }^{2}}+R_{\mathrm{p}}{ }^{3}\right]+\left(L-R_{p}\right) \pi R_{\mathrm{p}}{ }^{2} .
\end{aligned}
$$

The sphericity of the cell is defined as:

$\mathrm{Sph}=4.836 V^{2 / 3} / A$.

To avoid bias in selecting cells to characterize a particular population, an arbitrary field of view was chosen and every cell in the field was measured.

Statistics. The standard error (SE) for each sample (patient and control) was calculated from the standard deviation of the sample (SD):

$$
\mathrm{SE}=\mathrm{SD} / \sqrt{\mathrm{n}-1},
$$

where $n$ is the sample size. The standard error of the ratio $(C=A / B)$ or of the product (in calculating $\eta)(C=A \cdot B)$ were obtained via the following approximate relationship:

$\left(\frac{S E_{C}}{\langle C\rangle}\right)^{2}=\left(\frac{S E_{A}}{\langle A\rangle}\right)^{2}+\left(\frac{S E_{B}}{\langle B\rangle}\right)^{2}$,

where \langle\rangle indicates the average value of the sample, product, or ratio.

\section{Results}

Blood samples from a total of 21 patients were examined (Table I), including one $(17,17 \mathrm{a})$ who was tested both pre- and postsplenectomy. Patients 19 and 20 are not spectrin deficient, but have an abnormal variant of the protein band 4.1. Patient 21 is hematologically normal, and represents a splenectomized control. The membrane spectrin density (spectrin/band 3 ratio), erythrocyte spectrin content (measured by radioimmunoassay), the membrane shear modulus, the creasing pressure and the whole cell recovery time constant were determined for each sample (Table II). A minimum of 10 different cells from each sample were tested for shear modulus and creasing pressure and at least 16 cells were tested for the recovery time constant determination. Results for each patient were normalized relative to a matched control population that had been drawn concurrently and shipped on ice with the abnormal samples. For shear modulus and creasing pressure measurement, controls and abnormal cells were measured with the same pipette, and controls were tested before and after the abnormal cells to insure that the condition of the cells and the pipette did not change during the course of the experiment. The results are summarized in Table II, which lists the normalized values of the relevant parameters for each patient. Standard errors of the ratios of the mechanical properties are listed in the table. Biochemical measurements were repeatable within $\pm 5.0 \%$ of the average for the spectrin/band 3 ratios and $\pm 7.0 \%$ for radioimmunoassay. The results for each mechanical parameter are shown as a function of spectrin/band 3 ratio or spectrin radioimmunoassay in Figs. 3-6.

Cellular morphology. Most of the patients in the present study had some cells that were morphologically "normal", i.e., they were biconcave disks of approximately the same diameter as controls. In general, the distribution of cell size was broader for the abnormal samples than for controls, and there were some cells too small or too spherical to be used for mechanical studies. For all but seven of the patients, $>80 \%$ of the cells were suitable for mechanical measurements. The exceptions include the most severely affected recessive patients and patient 18 , who was poikilocytic. For patients $1,2,6-8$, and 18 , 
Table I. Clinical and Genetic Analysis of Patients

\begin{tabular}{|c|c|c|c|c|c|}
\hline Family & Patient & Clinical severity & Splenectomy & Genetic or biochemical abnormality & Zygosity \\
\hline \multicolumn{6}{|c|}{ Recessive spherocytosis } \\
\hline \multirow[t]{4}{*}{ A } & 1 (Sister) & Nearly lethal & + & $\alpha$ II Spectrin variant & Homozygote \\
\hline & 2 (Sister) & Nearly lethal & + & $\alpha$ II Spectrin variant & Homozygote \\
\hline & 3 (Mother) & Asymptomatic & & $\alpha$ II Spectrin variant & Heterozygote (carrier) \\
\hline & 4 (Distant cousin) & Severe & + & $\alpha$ II Spectrin variant & Homozygote \\
\hline B & 5 (Male) & Nearly lethal & + & $\alpha$ Spectrin RFLP* & Homozygote \\
\hline $\mathrm{C}$ & 6 (Male) & Nearly lethal & + & $\alpha$ II Spectrin variant & Homozygote \\
\hline \multirow[t]{2}{*}{$\mathrm{D}$} & 7 (Brother) & Severe & + & $\alpha$ II Spectrin variant & Homozygote \\
\hline & 8 (Sister) & Moderate & + & $\alpha$ II Spectrin variant & Homozygote \\
\hline \multirow[t]{2}{*}{$\mathbf{E}$} & 9 (Sister) & Nearly lethal & + & Unidentified & Presumed homozygote \\
\hline & 10 (Brother) & Nearly lethal & + & Unidentified & Presumed homozygote \\
\hline \multicolumn{6}{|c|}{ Dominant spherocytosis } \\
\hline \multirow[t]{2}{*}{$\mathbf{F}$} & 11 (Mother) & Mild & + & Unidentified & Heterozygote \\
\hline & 12 (Son) & Mild & & Unidentified & Heterozygote \\
\hline G & 13 (Female) & Mild & + & Unidentified & Heterozygote \\
\hline \multirow[t]{2}{*}{$\mathbf{H}$} & 14 (Mother) & Moderate & + & Unidentified & Heterozygote \\
\hline & 15 (Son) & Moderate & & Unidentified & Heterozygote \\
\hline I & 16 (Female) & Moderate & + & Spectrin-4.1 assoc. & Heterozygote \\
\hline \multirow[t]{2}{*}{$\mathbf{J}$} & 17 (Female) & Moderate & & Unidentified & Heterozygote \\
\hline & 17A (Same pt.) & Moderate & + & Unidentified & Heterozygote \\
\hline \multicolumn{6}{|c|}{ Elliptocytosis } \\
\hline $\mathbf{K}$ & 18 (Female) & Moderate & + & $\begin{array}{l}\alpha \mathrm{IT} 68 \text { Spectrin variant (and } \\
\text { reduced ankyrin sites) }\end{array}$ & Homozygote \\
\hline \multirow[t]{2}{*}{$\mathbf{L}$} & 19 (Father) & Asymptomatic & & Elongated 4.1 variant & Heterozygote \\
\hline & 20 (Son) & Asymptomatic & & Elongated 4.1 variant & Heterozygote \\
\hline \multicolumn{6}{|c|}{ Asplenic control } \\
\hline $\mathbf{M}$ & 21 (Male) & Normal & + & None & \\
\hline
\end{tabular}

* RFLP, restriction fragment length polymorphism/homozygote with parental consanguinity. ${ }^{\dagger}$ Described in references $2,13,14$, and 35 .

only $7-20 \%$ of the cells were suitable for mechanical measurements, and for patient $4,40-60 \%$ of the cells were suitable for measurement. For these patients, selectivity in choosing cells for measurement may have resulted in average values for mechanical properties that are not representative of the total cell population (used for biochemical studies). This situation is regrettable, but because of the technical limitations of our measurements and the nature of the abnormal cell population, it is unavoidable. Nevertheless, if the selected cells are morphologically closer to normal than the average, it is very likely that measured viscoelastic coefficients actually underestimate the true extent of the mechanical abnormality. For most of the patients studied, it was possible to take a relatively unbiased sample of the population.

Sphericity. The primary morphological difference between HS cells and controls is increased sphericity. There is also a slight reduction in mean surface area for the HS cells, but because the distribution of areas within the populations is large, there is typically greater than $80 \%$ overlap in the distributions of area for normal and abnormal cells. This is illustrated in Fig. 2, which shows the sphericity plotted as a function of area for cells of patient 4 and the matched control.

Shear modulus. Striking correlations were observed when either membrane spectrin density (Fig. 3) or erythrocyte spectrin content (Fig. 3, inset) were plotted against shear modulus
$\left(P_{\mathrm{m}=0}<0.001\right) .^{2}$ These two methods for spectrin quantitation provide somewhat different information. Spectrin density (spectrin/band 3 ratio) is a unitless comparison of membrane spectrin and band 3, the latter being an index of membrane surface area. (Only about $15 \%$ of band 3 is tethered to the membrane skeleton [24].) The plot for all data points of spectrin density vs. shear modulus appears to be curvilinear (Fig. 3, dashed line). Sampling error was a concern for the least normal samples, and when data from the more normal samples were analyzed (spectrin/band $3>0.6$ ), a linear relationship was identified with $y$ intercept $=0.13$, and slope $=0.83(r$ $=0.79$ ). These compare well with the values anticipated if shear modulus is directly proportional to spectrin density, $y$ intercept $=0$, slope $=1$.

The correlation between erythrocyte spectrin content (determined by radioimmunoassay) and shear modulus is also notable (Fig. 3, inset), with spectrin content expressed as fraction of normal control. The correlation with the shear modulus is statistically convincing, yet the linear relationship has a $y$ intercept $=0.45$ and slope $=0.5$. Although erythrocyte spectrin content measurement by radioimmunoassay is a more reliable measurement than spectrin density, the spectrin con-

2. $P_{m=0}$ is the probability that the slope of the linear regression $(m)$ is zero, i.e., the probability that there is no correlation. 
Table II. Erythrocyte Membrane Spectrin Content and Material Properties

\begin{tabular}{|c|c|c|c|c|c|c|c|}
\hline Family & Patient & $\begin{array}{l}\text { Spectrin } \\
\text { radioimmunoassay }\end{array}$ & $\begin{array}{l}\text { Spectrin/band } 3 \\
\text { ratio }^{\ddagger}\end{array}$ & Shear modulus & Creasing pressure & $\begin{array}{l}\text { Recovery } \\
\text { time constant }\end{array}$ & Membrane viscosity \\
\hline & & fraction of control & fraction of control & $\mu / \mu_{o} \pm S E$ & $P_{d} / P_{c o} \pm S E$ & $t_{d} / t_{\infty} \pm S E$ & $\eta / \eta_{o} \pm S E$ \\
\hline \multicolumn{8}{|c|}{ Recessive spherocytosis } \\
\hline \multirow[t]{5}{*}{ A } & 1 & 0.34 & 0.54 & $0.62 \pm 0.04$ & $0.72 \pm 0.04$ & $0.62 \pm 0.07$ & $0.39 \pm 0.05$ \\
\hline & 2 & 0.31 & 0.52 & $0.62 \pm 0.03$ & $0.67 \pm 0.03$ & $0.76 \pm 0.07$ & $0.47 \pm 0.05$ \\
\hline & 3 & 0.98 & 0.97 & $0.82 \pm 0.09$ & $0.78 \pm 0.04$ & - & - \\
\hline & 4 & 0.59 & 0.68 & $0.73 \pm 0.05$ & $0.58 \pm 0.04$ & $0.85 \pm 0.06$ & $0.62 \pm 0.06$ \\
\hline & & & & & & $0.85 \pm 0.05$ & $0.66 \pm 0.05$ \\
\hline B & 5 & 0.45 & 0.73 & $0.77 \pm 0.04$ & $0.77 \pm 0.03$ & $0.85 \pm 0.05$ & $0.66 \pm 0.05$ \\
\hline $\mathrm{C}$ & 6 & 0.33 & 0.55 & $0.61 \pm 0.04$ & $0.64 \pm 0.03$ & $0.87 \pm 0.07$ & $0.53 \pm 0.06$ \\
\hline \multirow[t]{2}{*}{ D } & 7 & 0.51 & 0.65 & $0.61 \pm 0.03$ & $0.66 \pm 0.02$ & $0.76 \pm 0.04$ & $0.47 \pm 0.03$ \\
\hline & 8 & 0.57 & 0.71 & $0.73 \pm 0.05$ & $0.66 \pm 0.03$ & $0.97 \pm 0.07$ & $0.71 \pm 0.07$ \\
\hline \multirow[t]{2}{*}{$\mathrm{E}$} & 9 & 0.34 & 0.43 & $0.62 \pm 0.09$ & $0.57 \pm 0.04$ & $0.69 \pm 0.04$ & $0.43 \pm 0.07$ \\
\hline & 10 & 0.33 & 0.43 & $0.63 \pm 0.07$ & $0.53 \pm 0.04$ & $0.62 \pm 0.05$ & $0.39 \pm 0.05$ \\
\hline \multicolumn{8}{|c|}{ Dominant spherocytosis } \\
\hline \multirow[t]{2}{*}{$\mathbf{F}$} & 11 & 0.82 & 0.82 & $0.89 \pm 0.07$ & $0.73 \pm 0.04$ & $0.73 \pm 0.06$ & $0.65 \pm 0.08$ \\
\hline & 12 & 0.77 & 0.89 & $0.82 \pm 0.04$ & $0.71 \pm 0.03$ & $0.82 \pm 0.07$ & $0.67 \pm 0.06$ \\
\hline G & 13 & 0.75 & 0.88 & $0.92 \pm 0.04$ & $0.92 \pm 0.05$ & $1.18 \pm 0.09$ & $1.09 \pm 0.09$ \\
\hline \multirow[t]{2}{*}{$\mathbf{H}$} & 14 & 0.69 & 0.80 & $0.72 \pm 0.04$ & $0.67 \pm 0.03$ & $1.37 \pm 0.12$ & $0.98 \pm 0.10$ \\
\hline & 15 & $0.68^{*}$ & 0.86 & $0.70 \pm 0.04$ & $0.69 \pm 0.04$ & $1.03 \pm 0.09$ & $0.72 \pm 0.08$ \\
\hline I & 16 & $0.66^{*}$ & 0.80 & $0.83 \pm 0.08$ & $0.75 \pm 0.07$ & $0.75 \pm 0.16$ & $0.62 \pm 0.15$ \\
\hline \multirow[t]{2}{*}{$\mathbf{J}$} & 17 & 0.81 & 0.93 & $0.90 \pm 0.05$ & $0.85 \pm 0.03$ & $0.92 \pm 0.07$ & $0.82 \pm 0.08$ \\
\hline & $17 \mathrm{~A}$ & 0.80 & 0.90 & $0.82 \pm 0.05$ & $0.83 \pm 0.04$ & $0.82 \pm 0.05$ & $0.67 \pm 0.06$ \\
\hline \multicolumn{8}{|c|}{ Elliptocytosis } \\
\hline $\mathrm{K}$ & 18 & $0.55^{*}$ & 0.70 & $0.72 \pm 0.10$ & $0.63 \pm 0.05$ & $1.06 \pm 0.14$ & $0.76 \pm 0.15$ \\
\hline \multirow[t]{2}{*}{$\mathbf{L}$} & 19 & $1.0^{*}$ & 0.99 & $0.97 \pm 0.06$ & $0.92 \pm 0.05$ & $0.66 \pm 0.03$ & $0.64 \pm 0.05$ \\
\hline & 20 & $1.0^{*}$ & 1.00 & $1.04 \pm 0.05$ & $1.03 \pm 0.05$ & $0.74 \pm 0.05$ & $0.77 \pm 0.06$ \\
\hline \multicolumn{8}{|c|}{ Asplenic control } \\
\hline & 21 & 1.06 & 0.89 & $0.99 \pm 0.03$ & $0.92 \pm 0.06$ & $1.03 \pm 0.07$ & $1.02 \pm 0.07$ \\
\hline
\end{tabular}

* Spectrin content from five individuals was determined from the normalized ratio of spectrin relative to band 3 (sp/b3) eluted with $25 \%$ (vol/ vol) pyridine $/ \mathrm{H}_{2} \mathrm{O}$ from Coomassie Blue stained SDS-PAGE slabs. Over-estimation of spectrin was corrected by comparing the sp/b3 with the correlation of $\mathrm{sp} / \mathrm{b} 3$ to spectrin content as measured by radioimmunoassay (5). ${ }^{\ddagger}$ RIA measurements were repeatable within $7.0 \%$ of the average value. Spectrin/band 3 measurements were repeatable within $5.0 \%$ of the average value.

tent does not reflect any secondary loss of unsupported membrane (containing phospholipid and untethered band 3 ). It has been shown with a band 3 radioimmunoassay that erythrocytes with gross deficiencies of spectrin have smaller, presumably secondary deficiencies of band 3 as well as minor reductions of other membrane proteins (4). Measurements of cell surface area using micropipettes indicate that the average surface area of cells from HS patients $4,5,8,11-14$ was up to $10 \%$ less than control. Thus, the reduction in spectrin content for the HS membranes is in part compensated by reductions of membrane surface area, so that the surface density of spectrin on the membrane is reduced to an intermediate level. For this reason, we expect reductions in the spectrin density (spectrin/ band 3 ratio) to correspond more directly to reductions in the elastic shear modulus.

Important controls were included in these studies. Splenectomy has a small effect upon the spectrin/band 3 ratio, but this did not appear to alter the shear modulus when measured in an individual before and after splenectomy (patient 17, 17a), or in a normal individual who had undergone splenectomy following trauma (patient 21). Likewise, when assessed by percentage of circulating reticulocytes, mean cell age did not appear to have important effects either on the biochemical ab- normality or on membrane elasticity: similar shear moduli were measured in the splenectomized and unsplenectomized patients in families $\mathrm{F}$ and $\mathrm{H}$ as well as patient 17. Furthermore, the relationship between spectrin density and shear modulus is not unique for HS. Individuals with elliptocytosis resulting from an elongated protein 4.1 variant (patients 19 and 20) and an individual homozygous for an abnormal alpha I spectrin variant and intermediate spectrin deficiency (patient 18) all fell close to the profile for the HS patients.

Finally, neither an increase in sphericity nor a decrease in surface area has an effect on the measured value of the shear modulus. Populations of normal cells suspended in normal (295 mosmol PBS) and hypotonic (220 mosmol PBS) buffers had different sphericities $(0.80$ at 295 mosmol and 0.87 at 220 mosmol, $\bar{P}<0.001),{ }^{3}$ but exhibited similar shear moduli: at 295 mosmol, $\langle\mu\rangle=0.0044, \mathrm{SD}=0.0005, n=22$; at 220 mosmol, $\langle\mu\rangle=0.0043, \mathrm{SD}=0.0006, n=14$ (not significantly different by Student's $t$ test). Similarly, when large normal cells $\left(A>150 \mu \mathrm{m}^{2}\right)$ were compared with small normal cells $(A$ $<135 \mu \mathrm{m}^{2}$ ), there was also no significant difference in the

3. $\bar{P}$ is the probability that the means of the two populations are equal (compared to not equal) calculated from the Student's $t$ distribution. 


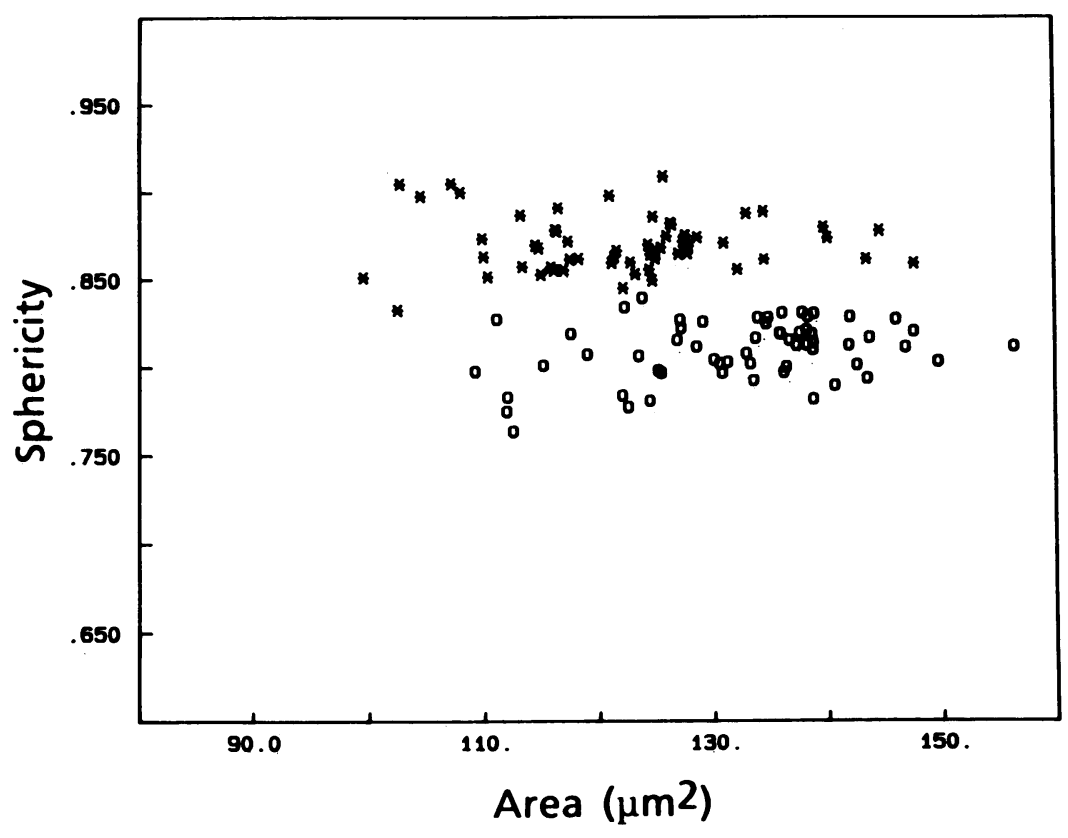

Figure 2. Sphericity plotted as a function of area for the cells of patient $4(*)$ with matched controls $(0)$. Note that although the mean area of the HS population is significantly less than control there is considerable overlap in surface area distribution between the two populations. Also note that the sphericity of the HS population is distinctly higher than control. $\langle\mathrm{A}\rangle_{\mathrm{HS}}=122 \mu \mathrm{m}^{2}, \mathrm{SD}=10, n=59 ;\langle\mathrm{A}\rangle_{\text {control }}=132$ $\mu \mathrm{m}^{2}, \mathrm{SD}=10, \mathrm{n}=60 ; \bar{P}<0.001 .\langle\mathrm{Sph}\rangle_{\mathrm{HS}}=0.87$, $\mathrm{SD}=0.02, n=59 ;\langle\mathrm{Sph}\rangle_{\text {oontrol }}=0.81, \mathrm{SD}=0.02, n$ $=60 ; \bar{P}<0.001$. mean shear moduli. For large cells $\left(\langle A\rangle=158 \mu \mathrm{m}^{2}\right),\langle\mu\rangle$ $=0.0043, \mathrm{SD}=0.0007, n=10$; for small cells $(\langle A\rangle=129$ $\left.\mu \mathrm{m}^{2}\right),\langle\mu\rangle=0.0044, \mathrm{SD}=0.0005, n=16$. Thus, the differences between abnormal and control populations that we have observed are not the result of differences in cell geometry, but reflect differences in the intrinsic properties of the membrane.

Creasing pressure. The pressure at which cells were observed to crease also decreased with membrane spectrin density (see Fig. 4). Because of severe shape abnormalities, the observed reduction in creasing pressure for patients $1,2,6,9$, 10 , and 18 may be due, in part, to geometric differences between the two cell populations, and may not be completely attributable to abnormally low bending stiffness.

Recovery time constant and membrane viscosity. The results for the recovery time constant and membrane viscosity are shown in Figs. 5 and 6. For most of the patients, the recovery time constant was reduced compared with control. Consequently, the membrane viscosity (equal to the product of the shear modulus and the time constant) is reduced even more than the shear modulus. This result indicates that the dependence of membrane viscosity on spectrin density is of order greater than one. Furthermore, it appears that the contribution of shear modulus (Fig. 3) is considerably more important than the contribution of recovery time constant (Fig. 5) to the observed correlation between reduced spectrin content and reduced membrane viscosity.

\section{Discussion}

Shear modulus. The similarity between the reduction in shear modulus and reductions in spectrin density and content is particularly interesting considering the thermodynamic meaning of the shear modulus. Elastic deformation of a surface requires energy, which is stored by the constituent molecules and recovered (or dissipated) during the return to the undeformed state. The shear modulus is a measure of the energy needed to deform the surface, per unit area, per unit deformation $(6,12)$. In general, this energy depends on the intrinsic stiffness of the molecules, the magnitude of the conformational change, and the number of molecules in the surface. The intrinsic molecular stiffness depends ultimately on the primary structure of the protein; the magnitude of the conformational change depends on the nature of the deformation and network topology; and the density of molecules on the surface should be reflected by the spectrin/band 3 ratio. The present results indicate that $(i)$ spectrin is the main energy storing molecule on the red cell membrane for elastic shear deformation, and (ii) in general, the intrinsic molecular stiffness and network topology are not significantly affected by the disorders, and the shear modulus depends simply on the surface density of spectrin in the membrane. These conclusions are consistent with the ultrastructural observations of Liu et al. (25) indicating that although the number of spectrin tetramers per actin protofilament is reduced in spectrin-deficient membranes, the normal organization of the skeleton (protofilaments of actin joined by spectrin oligomers) is found.

Data for the patients with the most severe spectrin deficiencies show less than expected reductions in shear modulus. As we mentioned in Results, this may be due to the necessity to select cells for measurement from a relatively small fraction of the total cell population because of the extensive geometric abnormalities found in these samples. It is interesting that the smallest values measured for the shear modulus are $\sim 60 \%$ of control, regardless of the reduction in spectrin density for the total population. If we have selected cells with the highest spectrin content for our mechanical measurements, this suggests that a cell must have at least $60 \%$ of normal spectrin density to maintain an approximately "normal" geometry. However, gradient separation techniques have failed to identify populations of cells with higher spectrin densities (data not shown), and it is not known how broad the distribution of spectrin densities within a cell population may be.

Observation of reductions in bending stiffness similar to the reduction in spectrin/band 3 ratio was unexpected. The bending stiffness of the membrane arises from its large resistance to changes in surface area (see Evans and Skalak [6] for 


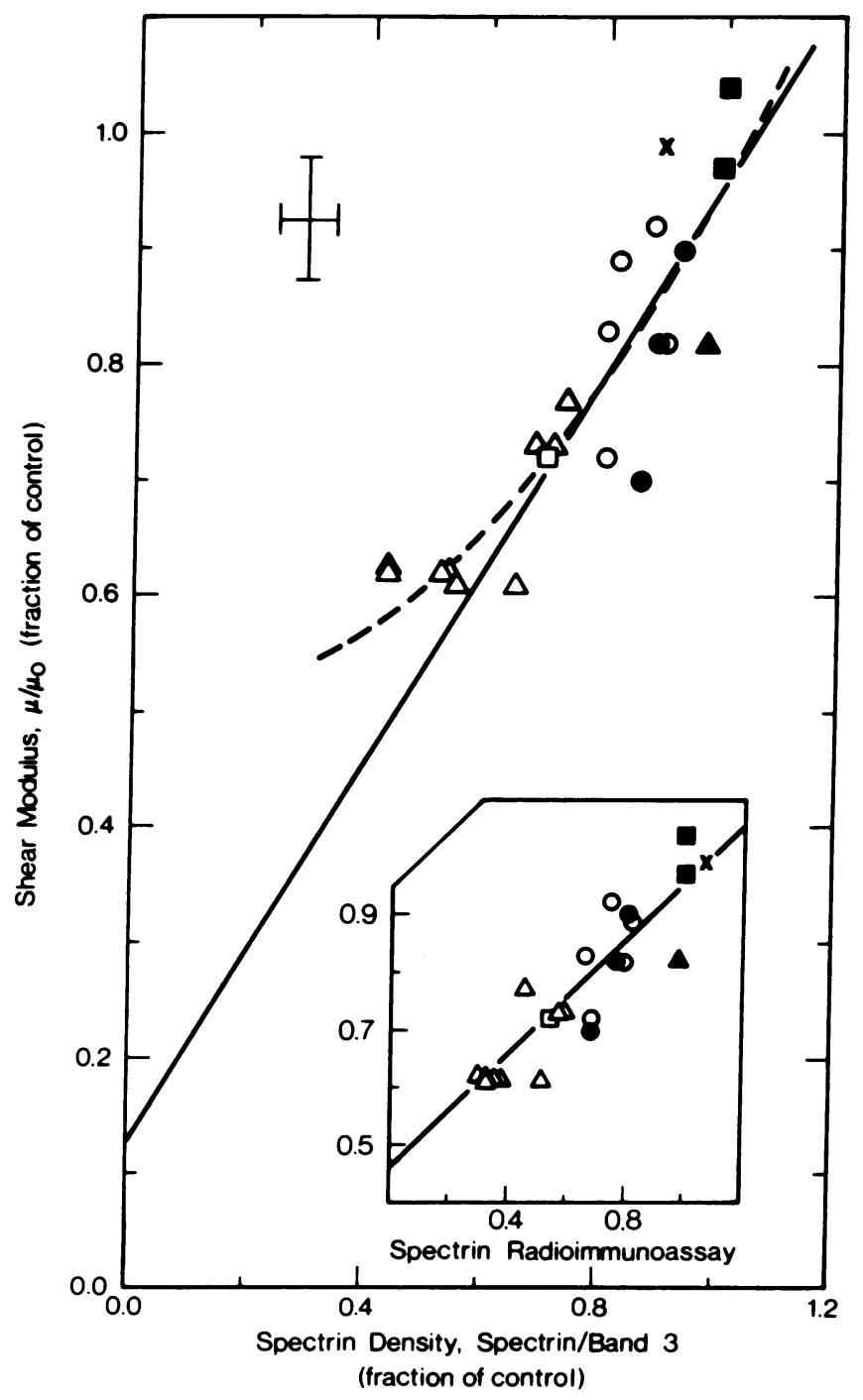

Figure 3. The relationship between spectrin density or spectrin content (inset) and the membrane shear modulus. Values are expressed as fractions of matched controls. Data for different abnormalities are coded as follows: $\Delta$ recessive $H S$, splenectomy; $\Delta$ recessive $H S$, heterozygote; $\bigcirc$ dominant HS, splenectomy; • dominant HS; $\square$ elliptocytosis, $\alpha$ spectrin variant; $\approx$ elliptocytosis, 4.1 variant; $\times$ control, splenectomy. The solid line is the linear regression to the data for spectrin density greater than 0.6: $y$ intercept $=0.13$, slope $=0.83, r$ $=0.79, P_{m=0}<0.001, n=17$. Dashed curve shows curvilinear (quadratic) appearance of the dependence of shear modulus on spectrin density when all data are included. The cross at the upper left indicates plus or minus the average standard error for the shear modulus ratio and the confidence interval $( \pm 0.05)$ for the spectrin/band 3 ratio. For spectrin content (inset) $y$ intercept $=0.45$, slope $=0.50, r$ $=0.90, P_{m=0}<0.001, n=22$.

details). Recent observations by Evans and Needham (26) of the area compressibility of cholesterol-containing lipid bilayers indicate that much or all of the resistance of the red cell membrane to area change might be attributable to the membrane bilayer. Furthermore, it is intuitively unlikely that an elastomeric structure such as the membrane skeleton, that has such a small resistance to extensional deformation, could contribute significantly to the resistance to area change, which is characterized by a modulus that is four to five orders of magnitude

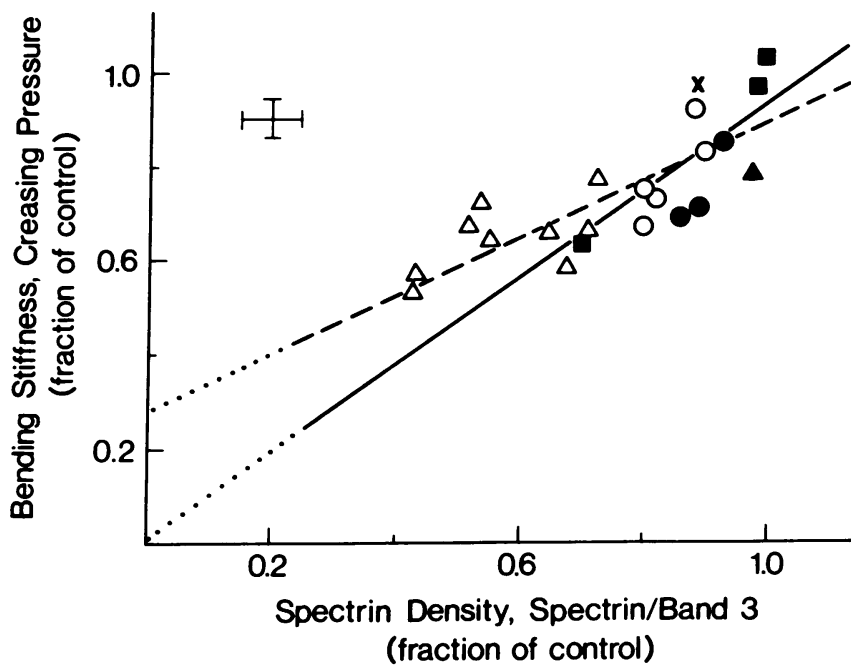

Figure 4. Relationship between spectrin density and creasing pressure (bending stiffness). Solid line shows linear regression to samples not likely to be affected by geometric abnormalities (normalized spectrin density $>0.6$ ). The $y$ intercept $=0.03$, slope $=0.89, r$ $=0.79, P_{m=0}<0.001, n=17$. Dashed line is the linear regression to all of the data: $y$ intercept $=0.30$, slope $=0.58, r=0.79, P_{m=0}$ $<0.001, n=22$. Data symbols are defined in the legend of Fig. 3 . The cross at the upper left indicates plus or minus the average standard error for the creasing pressure ratios and the confidence interval for the spectrin/band 3 ratio.

larger than the membrane shear modulus (500 dyn/cm compared with $0.007 \mathrm{dyn} / \mathrm{cm}$ ). One possible explanation for our observations is that the spectrin may alter the arrangement or composition of the bilayer. A preliminary report documents abnormal phospholipid organization in membranes from a patient with gross spectrin deficiency (27). Furthermore,

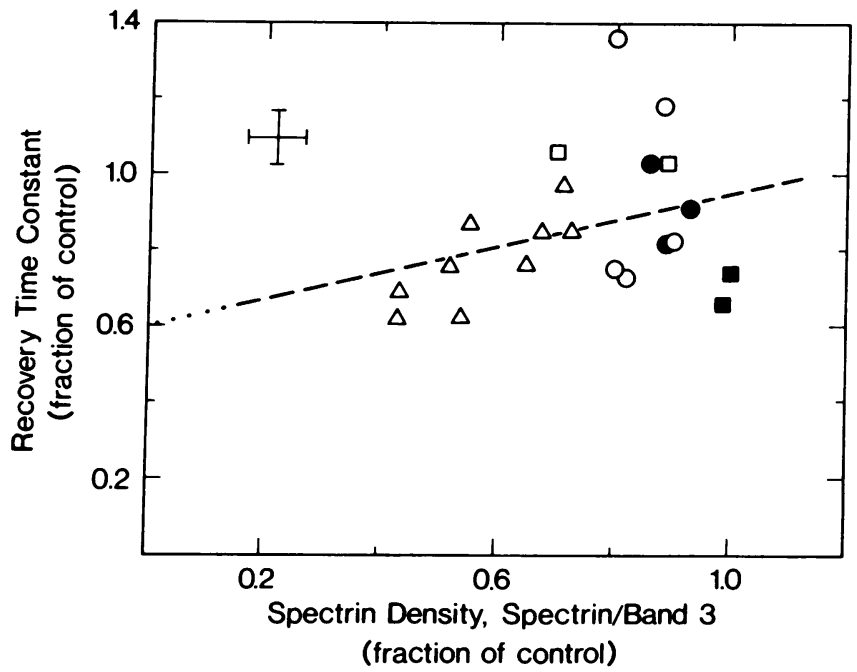

Figure 5. Relationship between spectrin density and recovery time constant. Data symbols are defined in the legend of Fig. 3. The dashed line shows a linear regression to the data, but there was no significant correlation between recovery time constant and spectrin density $(r=0.32)$. The cross at the upper left indicates plus or minus the average standard error for the time constant ratios and the confidence interval for the spectrin/band 3 ratio. 


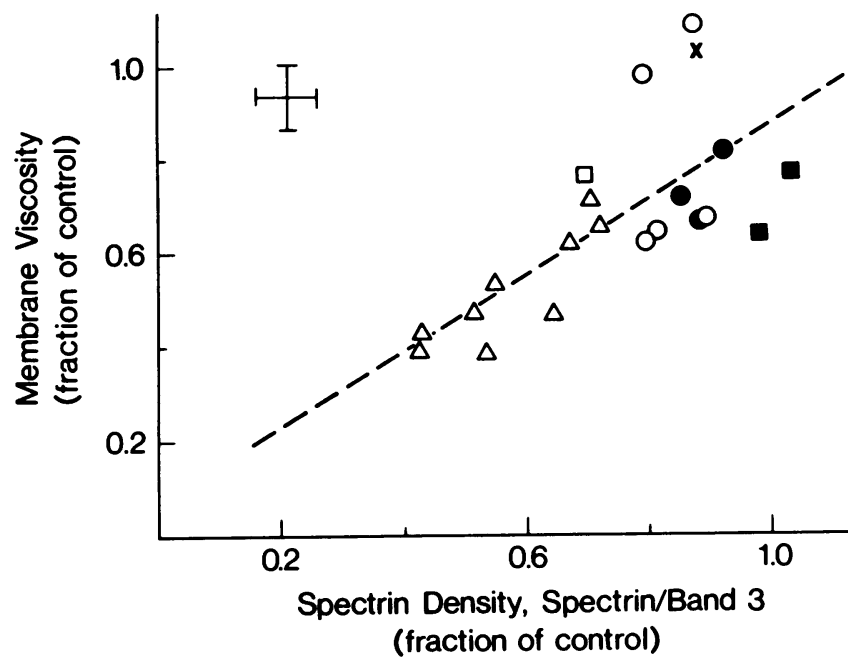

Figure 6. Relationship between spectrin density and membrane viscosity. Dashed line shows the linear regression to the data: $y$ intercept $=0.08$, slope $=0.79, r=0.71, P_{m=0}<0.001, n=21$. For data with a normalized spectrin density $>0.6$, no significant correlation was observed ( $r=0.35$, curve not shown). Data symbols are defined in the legend of Fig. 3. The cross at the upper left shows plus or minus the average standard error of the viscosity ratios and the confidence interval for the spectrin/band 3 ratio.

Evans and Needham (26) have observed that the area modulus of cholesterol-containing bilayers is reduced when protein is added to the membrane, indicating that the presence of protein may alter the partitioning of the cholesterol/phospholipid in the surface. Thus, it appears likely that the apparent reduction in bending stiffness that we have observed is due to an indirect effect of spectrin on the organization or composition of the membrane bilayer.

The lack of effect of abnormality in band 4.1 on membrane bending stiffness and shear elasticity demonstrates an important distinction between the function of spectrin and the function of band 4.1. Takakuwa and co-workers (28) have demonstrated an important role for band 4.1 in maintaining red cell membrane stability under large shear stresses. The present results indicate that an abnormal protein 4.1 variant resulting in elliptocytosis did not contribute measurably to shear elasticity when the forces on the cell are small compared to forces needed to cause membrane fragmentation. Thus, spectrin appears to determine the membrane response to small forces, but band 4.1 contributes to the stability of the membrane when it is subjected to large forces.

It is interesting that the membrane viscosity for these elliptocytic membranes is lower than normal (see Table II, patients 19 and 20). This observation is somewhat surprising because the variant band 4.1 is larger than the normal molecule, and a larger molecule might be expected to increase cellular viscosity. At the present time, however, it is not known if the number of copies of the variant molecule is normal. (A decrease in number of molecules would be expected to reduce membrane viscosity.)

The membrane viscosity of the spectrin-deficient cells did not follow the same pattern of variation as did the membrane shear modulus or the bending stiffness. Generally, the viscosity was more markedly reduced than the shear modulus, although this was not always the case. These observations might have been expected for two reasons: first, the dependence of viscosity on protein concentration is expected to be nonlinear according to molecular theories of viscosity. In these molecular theories (29) the viscosity depends not only on the density of molecules, but also on the number of entanglements between molecules (which increases with molecular concentration). Thus, the viscosity of the membrane is expected to depend on spectrin density raised to a power greater than one. Second, there is evidence that the membrane viscosity is more sensitive to environmental factors than the shear modulus. For example, the temperature dependence of the viscosity is much larger than the temperature dependence of the shear modulus $(30,31)$.

Of more relevance than temperature variation, is the observation that the viscosity depends strongly on intracellular hemoglobin concentration (MCHC) $(32,33)$. Because of this, differences in cell age might affect our observations of membrane viscosity. Because "young" cells are thought to be less dense (i.e., have a lower MCHC), and because spectrin-deficient cells are likely to have shortened lifetimes resulting in a relatively "young" population of cells, it is possible that part of the reduction in viscosity we have observed may be due to the youth of the abnormal cell populations. On the other hand, loss of membrane surface in HS characteristically increases the MCHC, which would raise the viscosity. In any case, it should be emphasized that neither the spectrin content nor the membrane shear modulus are appreciably affected by cell age ( 5 , 34 ), and the differences in shear modulus between normal and abnormal populations are not attributable to differences in cell age.

Single-cell, micropipette experiments are designed to assess the intrinsic material properties of the membrane. Slight geometric differences between cells are not expected to affect the outcome of the measurements. Our observation that the measured value of the shear modulus of normal cells is not affected by differences in cell surface area or sphericity confirm this expectation. Thus, the differences we have observed between normal and abnormal populations of cells reflect differences in the intrinsic properties of the membranes, and are not the result of differences in cellular geometry.

The present study documents the effect of spectrin deficiency on the viscoelastic properties of erythrocyte membrane. Similarities between the fractional reduction in shear modulus and the fractional reduction in spectrin/band 3 suggest that, in general, membrane shear elasticity is directly proportional to the surface density of spectrin on the membrane. The apparent membrane bending stiffness also appears to be reduced in proportion to the density of spectrin, but the prevailing view that bending stiffness arises from the membrane bilayer leads us to propose that the reduction in bending stiffness may be due to an indirect effect of spectrin on the composition and/or molecular organization of the membrane bilayer. The membrane viscosity is also reduced relative to control, but the nature of the relationship between spectrin density and membrane viscosity is not clearly delineated.

\section{Acknowledgments}

The authors would like to thank the patients and their families who cooperated to make this study possible. We would also like to thank Dr. Campbell McMillan for his assistance in obtaining blood samples and Dr. Pamela Becker for obtaining blood samples and discussing her 
own data with us prior to publication. The authors recognize the technical support of Richard Bauserman, Donna Brooks and Gary Erwin.

This work was supported under grants HL-31524 (Dr. Waugh) and HL-33391 (Dr. Agre) from the U. S. Public Health Service.

\section{References}

1. Weed, R. I. 1975. Hereditary spherocytosis. A review. Arch. Intern. Med. 135:1316-1323.

2. Becker, P. S., and S. E. Lux. 1985. Hereditary spherocytosis and related disorders. Clin. Hematol. 14:15-43.

3. Bennett, V. 1985. The membrane skeleton of human erythrocytes and its implication for more complex cells. Annu. Rev. Biochem. 54:273-304.

4. Agre, P., J. F. Casella, W. H. Zinkham, C. McMillan, and V. Bennett. 1985. Partial deficiency of spectrin in hereditary spherocytosis. Nature (Lond.). 314:380-383.

5. Agre, P., A. Asimos, J. F. Casella, and C. McMillan. 1986. Inheritance pattern and clinical response to splenectomy as a reflection of erythrocyte spectrin deficiency in hereditary spherocytosis. $N$. Engl. J. Med. 315:1579-1583.

6. Evans, E. A., and R. Skalak. 1979. Mechanics and thermodynamics of biomembrane. CRC Crit. Rev. Bioengr. 3:181-418.

7. Hochmuth, R. M., and R. E. Waugh. 1987. Erythrocyte membrane elasticity and viscosity. Annu. Rev. Physiol. In press.

8. Chien, S., K. L. P. Sung, R. Skalak, S. Usami, and A. Tozeren. 1978. Theoretical and experimental studies on viscoelastic properties of erythrocyte membrane. Biophys. J. 24:463-487.

9. Tozeren, A., R. Skalak, B. Fedorciw, K. L. P. Sung, and S. Chien. 1984. Constitutive equations of erythrocyte membrane incorporating evolving preferred configuration. Biophys. J. 45:541-549.

10. Waugh, R. E. 1983. Effects of abnormal cytoskeletal structure on erythrocyte membrane mechanical properties. Cell Motility. 3:609-622.

11. Marchesi, S. L., J. T. Letsinger, and V. T. Marchesi. 1985. Localization of a third $\alpha$-spectrin abnormality in hereditary elliptocytosis to the $\alpha \mathrm{I} 80 \mathrm{kD}$ domain. Purification and characterization of all three abnormal domains. Blood. 66:36a. (Abstr.)

12. Waugh, R. E. 1987. Effects of inherited membrane abnormalities on the viscoelastic properties of erythrocyte membrane. Biophys. $J$. 51:363-369.

13. Conboy, J., Y. W. Kan, P. Agre, and N. Mohandas. 1986. Molecular characterization of hereditary elliptocytosis due to an elongated protein 4.1. Blood. 68:34a. (Abstr.)

14. Letsinger, J. T., P. Agre, and S. L. Marchesi. 1986. High molecular weight protein 4.1 in the cytoskeletons of hereditary elliptocytes. Blood. 68:38a. (Abstr.)

15. Bennett, V. 1983. Proteins involved in membrane-cytoskeleton association in human erythrocytes: spectrin, ankyrin and band 3 . Methods Enzymol. 96:313-324.

16. Fairbanks, G., T. L. Steck, and D. F. H. Wallach. 1971. Electrophoretic analysis of the major polypeptides of the human erythrocyte membrane. Biochemistry. 10:2606-2617.

17. Fenner, C., R. R. Traut, D. T. Mason, and J. Wikman-Coffelt.
1975. Quantification of Coomassie blue stained proteins in polyacrylamide gels based on analysis of eluted dye. Anal. Biochem. 63:595602.

18. Hunter, W. M., and F. C. Greenwood. 1962. Preparation of Iodine-131 labelled human growth hormone of high specific activity. Nature (Lond.). 194:495-496.

19. O'Keefe, E., and V. Bennett. 1980. Use of immunoglobulinloaded protein A bearing staphylococci as a primary solid phase immunoadsorbent in radioimmunoassay. J. Biol. Chem. 255:561-568.

20. Evans, E. A., and P. L. La Celle. 1975. Intrinsic material properties of the erythrocyte membrane indicated by mechanical analysis of deformation. Blood. 45:29-43.

21. Waugh, R. E. 1986. Effects of 2,3-diphosphoglycerate on the mechanical properties of erythrocyte membrane. Blood. 68:231-238.

22. Evans, E. A. 1983. Bending elastic modulus of red blood cell membrane derived from buckling instability in micropipet aspiration tests. Biophys. J. 43:27-30.

23. Hochmuth, R. M., P. R. Worthy, and E. A. Evans. 1979. Red cell extensional recovery and the determination of membrane viscosity. Biophys. J. 26:101-114.

24. Bennett, V. 1982. Isolation of an ankyrin-band 3 oligomer from human erythrocyte membranes. Biochim. Biophys. Acta. 689:475484.

25. Liu, S. C., L. H. Derick, P. C. Agre, and J. Palek. 1986. Alteration of the red cell membrane skeletal ultrastructure in hereditary spherocytosis. Blood. 68:56a (Abstr.).

26. Evans, E., and D. Needham. 1986. Giant vesicle bilayers composed of mixtures of lipids, cholesterol and polypeptides. Thermomechanical and (mutual) adherance properties. Faraday Discuss. Chem. Soc. In press.

27. Lubin, B., D. Chiu, R. S. Schwartz, B. Cooper, K. John, L. Wolfe, and S. E. Lux. 1983. Abnormal membrane phospholipid organization in spectrin-deficient human red cells. Blood. 62:34a. (Abstr.)

28. Takakuwa, Y., G. Tchernia, M. Rossi, M. Benabadji, and N. Mohandas. 1986. Restoration of normal membrane stability to unstable protein 4.1-deficient erythrocyte membranes by incorporation of purified protein 4.1. J. Clin. Invest. 78:80-85.

29. Meares, P. 1965. Polymers, Structure and Bulk Properties. Van Nostrand Reinhold Co., London. 347-365.

30. Hochmuth, R. M., K. L. Buxbaum, and E. A. Evans. 1980. Temperature dependence of the viscoelastic recovery of red cell membrane. Biophys. J. 29:177-182.

31. Waugh, R., and E. A. Evans. 1979. Thermoelasticity of red blood cell membrane. Biophys. J. 26:115-132.

32. Nash, G. B., and H. J. Meiselman. 1983. Red cell and ghost viscoelasticity. Effects of hemoglobin concentration and in vivo aging. Biophys. J. 43:63-73.

33. Evans, E., N. Mohandas, and A. Leung. 1984. Static and dynamic rigidities of normal and sickle erythrocytes. Major influence of cell hemoglobin concentration. J. Clin. Invest. 73:477-488.

34. Nash, G. B., and S. J. Wyard. 1981. Erythrocyte membrane elasticity during in vivo aging. Biochim. Biophys. Acta. 643:269-275.

35. Winkelmann, J. C., S. L. Marchesi, P. Watkins, A. J. Linnenbach, P. Agre, and B. G. Forget. 1986. Recessive hereditary spherocytosis is associated with an abnormal alpha spectrin subunit. Clin. Res. 34:474a. (Abstr.) 